\title{
LAPTH
}

Laboratoire d'Annecy-le-Vieux de Physique Théorique

\section{Integrable systems with impurity}

\author{
E. Ragoucy* \\ LAPTH 9 Chemin de Bellevue, BP 110 F-74941 Annecy-le-Vieux Cedex, \\ France
}

\begin{abstract}
:
After reviewing some basic properties of RT algebras, which appear to be the natural framework to deal with integrable systems in presence of an impurity, we show how any integrable system (including these possessing translation invariance) can be promoted to an integrable system with an impurity which can reflect and transmit particles. The technics allows bulk translation invariant $S$-matrices while avoiding the no-go theorem stated recently about these laters.
\end{abstract}

Presented at the Vth International workshop on Lie theory and its applications in physics, Varna (Bulgaria), June 16-22, 2003.

January 2004

LAPTH-Conf-1022/04 math-ph/0401024

*ragoucy@lapp.in2p3.fr

†UMR 5108 du CNRS associée à l'Université de Savoie 


\section{Introduction}

In the realm of factorized scattering theories in integrable models on the line, the Zamolodchikov-Faddeev (ZF) algebra [1, 2] plays a fundamental role. It encodes (quadratically) the two-body scattering, which is the only particle interaction present in this case. There have been various generalizations, preserving integrability, to the case with a purely reflecting boundary [3, 4, 5, 6] or to the case of point-like impurities [7, 8]. In the later context, the relevant algebraic structure emerging recently [9, 10, 11, is the so-called reflectiontransmission (RT) algebra. It captures both the two-body scattering in the bulk, and the particle interaction with the impurity.

After reviewing some basic properties of RT algebras, we present a procedure which allows, starting from any integrable model on the line, to built an integrable model with a point-like impurity. In particular, despite the property stated in [8], we show that the present technics produces theories possessing a (non trivial) translation invariant scattering matrix in the bulk together with an impurity which reflects and transmits particles.

\section{An example of reflection-transmission al- gebra}

To sustain the introduction of RT algebra in the study of quantum impurity problems, we start with the simple example of (free) non-relativistic bosonic particles on $\mathbb{R}$, which interact with a $\delta$-type impurity localized in the origin. This model is well-known to be exactly solvable (see e.g. [12]). It has been presented from the RT algebra point of view in [10, so that we just sketch the results, refering to the original paper for more details. In particular, we will consider here only the case $\eta \geq 0$.

It is sufficient to investigate the spectral problem associated with the one-particle Hamiltonian

$$
H^{(1)}=\sum_{i=1}^{n}-\frac{1}{2} \partial_{x_{i}}^{2}+\eta \delta\left(x_{i}\right), \quad \eta \in \mathbb{R},
$$

defined on a suitable domain $\mathcal{D}_{\eta}$ of continuous functions on $\mathbb{R}$, which are twice differentiable in $\mathbb{R} \backslash\{0\}$ and satisfy

$$
\lim _{x \rightarrow 0^{+}}\left[\left(\partial_{x} \psi\right)(x)-\left(\partial_{x} \psi\right)(-x)\right]=2 \eta \psi(0) .
$$

$H^{(1)}$ is self-adjoint on $\mathcal{D}_{\eta}$. A set $\Phi=\left\{\psi_{k}^{ \pm}(x) ; k \in \mathbb{R}\right\}$ of orthogonal (generalized) eigenstates, verifying (2.2), is provided by:

$$
\psi_{k}^{ \pm}(x)=\theta(\mp k)\left\{\theta(\mp x) T(\mp k) e^{i k x}+\theta( \pm x)\left[e^{i k x}+R(\mp k) e^{-i k x}\right]\right\},
$$


where $\theta$ denotes the standard Heaviside function and

$$
T(k)=\frac{k}{k+i \eta}, \quad R(k)=\frac{-i \eta}{k+i \eta} .
$$

The family $\bar{\Phi}=\left\{\bar{\psi}_{-k}^{ \pm}(x) ; k \in \mathbb{R}\right\}$, where the bar stands for complex conjugation, is also orthonormal. The systems $\Phi$ and $\bar{\Phi}$ represent physically scattering states and are separately complete in $L^{2}(\mathbb{R}, d x)$.

The reflection and transmission from the impurity give rise to a non-trivial scattering operator, which preserves the particle number. One can interpret $\psi_{k}^{ \pm}(x)$ asymptotically as incoming waves, traveling in $\mathbb{R}_{ \pm}$with momentum $k \neq 0$ towards the impurity. Accordingly, the vectors

$$
|k\rangle^{\text {in }}=\psi_{k}^{+}(x)+\psi_{k}^{-}(x) \text { and }|k\rangle^{\text {out }}=\bar{\psi}_{-k}^{+}(x)+\bar{\psi}_{-k}^{-}(x)
$$

form a basis of one-particle "in" states and "out" states resp. The oneparticle scattering operator is defined by $\mathbf{S}^{(1)}|k\rangle^{\text {out }}=|k\rangle^{\text {in }}$. By construction, $\mathbf{S}^{(1)}$ is a unitary operator on $L^{2}(\mathbb{R}, d x)$. The one-particle transition amplitude reads

$$
\begin{aligned}
{ }^{\text {out }}\langle p \mid k\rangle^{\text {in }}={ }^{\text {out }}\left\langle p\left|\mathbf{S}^{(1)}\right| k\right\rangle^{\text {out }=} & {[\theta(p) T(p)+\theta(-p) T(-p)] 2 \pi \delta(p-k) } \\
& +[\theta(p) R(p)+\theta(-p) R(-p)] 2 \pi \delta(p+k) .
\end{aligned}
$$

Thus, $R$ and $T$ indeed represent the transmission and reflection amplitudes.

The $n$-particle amplitude, with initial and final configurations satisfying $k_{1}<\ldots<k_{n}$ and $p_{1}>\ldots>p_{n}$ respect., can be expressed in terms of (2.6):

$$
\left.{ }^{\text {out }}\left\langle p_{1}, \ldots, p_{n} \mid k_{1}, \ldots, k_{n}\right\rangle\right\rangle^{\text {in }}=\prod_{i=1}^{n}{ }^{\text {out }}\left\langle p_{i} \mid k_{i}\right\rangle^{\text {in }} .
$$

The basic feature of RT algebra is to provide an algebraic framework for dealing with the above system when $\eta>0$, similar to the familiar canonical commutation approach, which works in the case $\eta=0$. We introduce the associative algebra $\mathcal{C}$ with identity element $\mathbf{1}$, generated by $\left\{a^{\dagger \xi}(k), a_{\xi}(k)\right.$ : $\xi= \pm, k \in \mathbb{R}\}$ obeying the commutation relations:

$$
\begin{aligned}
a_{\xi_{1}}\left(k_{1}\right) a_{\xi_{2}}\left(k_{2}\right)-a_{\xi_{2}}\left(k_{2}\right) a_{\xi_{1}}\left(k_{1}\right)= & 0 \\
a^{\dagger \xi_{1}}\left(k_{1}\right) a^{\dagger \xi_{2}}\left(k_{2}\right)-a^{\dagger \xi_{2}}\left(k_{2}\right) a^{\dagger \xi_{1}}\left(k_{1}\right)= & 0 \\
a_{\xi_{1}}\left(k_{1}\right) a^{\dagger \xi_{2}}\left(k_{2}\right)-a^{\dagger \xi_{2}}\left(k_{2}\right) a_{\xi_{1}}\left(k_{1}\right)= & {\left[\delta_{\xi_{1}}^{\xi_{2}}+\mathcal{T}_{\xi_{1}}^{\xi_{2}}\left(k_{1}\right)\right] 2 \pi \delta\left(k_{1}-k_{2}\right) \mathbf{1} } \\
& +\mathcal{R}_{\xi_{1}}^{\xi_{2}}\left(k_{1}\right) 2 \pi \delta\left(k_{1}+k_{2}\right) \mathbf{1}
\end{aligned}
$$

where

$$
\mathcal{T}(k)=\left(\begin{array}{cc}
0 & T(k) \\
\bar{T}(k) & 0
\end{array}\right), \quad \mathcal{R}(k)=\left(\begin{array}{cc}
R(k) & 0 \\
0 & \bar{R}(k)
\end{array}\right) .
$$


The right-hand side of eq. (2.10) captures the presence of the impurity. The term proportional to $\delta\left(k_{1}+k_{2}\right)$ reflects in particular the breaking of translation invariance due to the impurity. We will see that $\mathcal{C}$ is a particular RT algebra. For the moment we focus on the Fock representation $\mathcal{F}$ of $\mathcal{C}$, referring for the explicit construction to sect. 3.1. An essential feature of $\mathcal{F}$ is that the operators $a^{\dagger}(k)=\left(a^{\dagger+}(k), a^{\dagger-}(k)\right)$ and $a(k)=\left(\begin{array}{c}a_{+}(k) \\ a_{-}(k)\end{array}\right)$ in this representation satisfy

$$
\begin{aligned}
a(k) & =\mathcal{T}(k) a(k)+\mathcal{R}(k) a(-k), \\
a^{\dagger}(k) & =a^{\dagger}(k) \mathcal{T}(k)+a^{\dagger}(-k) \mathcal{R}(-k) .
\end{aligned}
$$

The relations (2.12), (2.13) encode the interaction with the impurity.

The vacuum state $\Omega \in \mathcal{F}$ obeys as usual $a_{\xi}(k) \Omega=0$. We denote by $(\cdot, \cdot)$ the scalar product in $\mathcal{F}$ and consider the vacuum expectation value

$$
\begin{aligned}
& \left(a^{\dagger \epsilon_{1}}\left(p_{1}\right) \ldots a^{\dagger \epsilon_{n}}\left(p_{n}\right) \Omega, a^{\dagger \xi_{1}}\left(k_{1}\right) \ldots a^{\dagger \xi_{n}}\left(k_{n}\right) \Omega\right), \\
& k_{1}<\cdots<k_{n}, \quad \xi_{i}=-\epsilon\left(k_{i}\right), \quad p_{1}>\cdots>p_{n}, \quad \epsilon_{i}=\epsilon\left(p_{i}\right)
\end{aligned}
$$

$\epsilon$ being the sign function. By means of eqs. (2.8)-(2.10) it is easily verified that (2.14) reproduces the amplitudes (2.7) for any $n$. Therefore, $\mathcal{C}$ provides a purely algebraic framework for constructing the scattering operator. In this framework, the Hamiltonian takes the form

$$
H=\frac{1}{2} \int_{-\infty}^{+\infty} \frac{d k}{2 \pi} k^{2} a^{\dagger \xi}(k) a_{\xi}(k) .
$$

The restriction of $H$ to the $n$-particle subspace is the algebraic counterpart of the starting Hamiltonian.

Thus, $\mathcal{C}$ is a universal and powerful tool for handling $\delta$-type impurities. One can view $\mathcal{C}$ as a central extension of the algebra of canonical commutation relations. A direct generalization is to substitute $\mathcal{T}(k) \mathbf{1}$ and $\mathcal{R}(k) \mathbf{1}$ in eq. (2.10) with new generators $t(k)$ and $r(k)$ which are no longer central. Moreover, in the spirit of the ZF algebra, it is possible to replace the bosonic exchange factor between $\left\{a^{\dagger}(k), a(k)\right\}$ with a more general one.

\section{The RT algebra}

Adopting throughout the paper the compact auxiliary spaces notation (see e.g. [13]), we define RT algebras [10] by:

Definition 3.1 (RT algebra $\mathcal{C}_{S}$ )

A RT algebra is generated by $\mathbf{1}$ and the generators $A(k), A^{\dagger}(k)$, (which are 
column and line vector valued resp.) $t(k)$ and $r(k)$ (which are matrix valued) obeying:

$$
\begin{aligned}
& A_{1}\left(k_{1}\right) A_{2}\left(k_{2}\right)= S_{21}\left(k_{2}, k_{1}\right) A_{2}\left(k_{2}\right) A_{1}\left(k_{1}\right) \\
& A_{1}^{\dagger}\left(k_{1}\right) A_{2}^{\dagger}\left(k_{2}\right)= A_{2}^{\dagger}\left(k_{2}\right) A_{1}^{\dagger}\left(k_{1}\right) S_{21}\left(k_{2}, k_{1}\right) \\
& A_{1}\left(k_{1}\right) A_{2}^{\dagger}\left(k_{2}\right)= A_{2}^{\dagger}\left(k_{2}\right) S_{12}\left(k_{1}, k_{2}\right) A_{1}\left(k_{1}\right)+\delta\left(k_{1}-k_{2}\right)\left(\delta_{12} \mathbf{1}+t_{12}\left(k_{1}\right)\right) \\
&+r_{12}\left(k_{1}\right) \delta\left(k_{1}+k_{2}\right) \\
& A_{1}\left(k_{1}\right) t_{2}\left(k_{2}\right)= S_{21}\left(k_{2}, k_{1}\right) t_{2}\left(k_{2}\right) S_{12}\left(k_{1}, k_{2}\right) A_{1}\left(k_{1}\right) \\
& A_{1}\left(k_{1}\right) r_{2}\left(k_{2}\right)= S_{21}\left(k_{2}, k_{1}\right) r_{2}\left(k_{2}\right) S_{12}\left(k_{1},-k_{2}\right) A_{1}\left(k_{1}\right) \\
& t_{1}\left(k_{1}\right) A_{2}^{\dagger}\left(k_{2}\right)= A_{2}^{\dagger}\left(k_{2}\right) S_{12}\left(k_{1}, k_{2}\right) t_{1}\left(k_{1}\right) S_{21}\left(k_{2}, k_{1}\right) \\
& r_{1}\left(k_{1}\right) A_{2}^{\dagger}\left(k_{2}\right)= A_{2}^{\dagger}\left(k_{2}\right) S_{12}\left(k_{1}, k_{2}\right) r_{1}\left(k_{1}\right) S_{21}\left(k_{2},-k_{1}\right) \\
& S_{12}\left(k_{1}, k_{2}\right) t_{1}\left(k_{1}\right) S_{21}\left(k_{2}, k_{1}\right) t_{2}\left(k_{2}\right)= \\
& t_{2}\left(k_{2}\right) S_{12}\left(k_{1}, k_{2}\right) t_{1}\left(k_{1}\right) S_{21}\left(k_{2}, k_{1}\right) \\
& S_{12}\left(k_{1}, k_{2}\right)
\end{aligned}
$$

where $r(k)$ and $t(k)$ satisfy:

$$
t(k) t(k)+r(k) r(-k)=\mathbb{I} \text { and } t(k) r(k)+r(k) t(-k)=0
$$

We refer to $r(k)$ and $t(k)$ as reflection and transmission generators. Here and below the $S$-matrix obeys the following well-known Yang-Baxter equation and unitarity relation:

$$
\begin{aligned}
& S_{12}\left(k_{1}, k_{2}\right) S_{13}\left(k_{1}, k_{3}\right) S_{23}\left(k_{2}, k_{3}\right)=S_{23}\left(k_{2}, k_{3}\right) S_{13}\left(k_{1}, k_{3}\right) S_{12}\left(k_{1}, k_{2}\right) \\
& S_{12}\left(k_{1}, k_{2}\right) S_{21}\left(k_{2}, k_{1}\right)=\mathbb{I} \otimes \mathbb{I}
\end{aligned}
$$

A special case of RT algebra is the boundary algebra $\mathcal{B}_{S}$ [14 defined as the coset of $\mathcal{C}_{S}$ by the relation $t(k)=0$. In the same way, $r(k)=0$ leads to an algebra with a purely transmitting impurity [15].

The generators $r(k)$ and $t(k)$ form a subalgebra $\mathcal{K}_{S} \subset \mathcal{C}_{S}$. This subalgebra contains itself two subalgebras: $\mathcal{R}_{S} \subset \mathcal{K}_{S}$, generated by $r(k)$, appears already in [4, called reflection algebra and $\mathcal{T}_{S} \subset \mathcal{K}_{S}$, generated by $t(k)$, called transmission algebra.

RT algebras possesses two important automorphisms: the (antilinear) adjunction $I$ given by

$$
I:\left\{\begin{array}{l}
a^{\dagger}(\chi) \mapsto a(\chi) \text { and } a(\chi) \mapsto a^{\dagger}(\chi) \\
r(\chi) \mapsto r(-\chi) \text { and } t(\chi) \mapsto t(\chi)
\end{array}\right.
$$


and the reflection-transmission automorphism:

$$
\varrho:\left\{\begin{array}{l}
a^{\dagger}(\chi) \mapsto a^{\dagger}(\chi) t(\chi)+a^{\dagger}(-\chi) r(\chi) \\
a(\chi) \mapsto t(\chi) a(\chi)+r(\chi) a(-\chi) \\
r(\chi) \mapsto r(\chi) \text { and } t(\chi) \mapsto t(\chi)
\end{array}\right.
$$

They both square to identity. We use them for the construction of Fock spaces for RT algebras.

\subsection{Fock spaces}

A Fock representation of the RT algebra with adjunction $\left\{\mathcal{C}_{S}, I\right\}$ is specified by the conditions:

(i) the representation space is a complex Hilbert space $\mathcal{H}$ with scalar product $(\cdot, \cdot)$;

(ii) the generators $\left\{a(\chi), a^{\dagger}(\chi), r(\chi), t(\chi)\right\}$ are operator-valued distributions with common and invariant dense domain $\mathcal{D} \subset \mathcal{H}$, where eqs. (3.1) - 3.11) hold;

(iii) the adjunction $I$ is realized as a conjugation with respect to $(\cdot, \cdot)$;

(iv) there exists a vacuum state $\Omega \in \mathcal{D}$, which is annihilated by $a(\chi)$. The vector $\Omega$ is cyclic with respect to $\left\{a^{\dagger}(\chi)\right\}$ and $(\Omega, \Omega)=1$.

There is a number of simple, but quite important consequences from the assumptions (i)-(iv). They are proved in [10].

Property 3.2 The reflection-transmission automorphism $\varrho$ is realized in any Fock representation by the identity operator.

Any RT algebra $\mathcal{C}_{S}$ admits in general a whole family $\mathcal{F}\left(\mathcal{C}_{S}\right)$ of Fock representations, parameterized by the vacuum expectation values

$$
\mathcal{R}(\chi)=(\Omega, r(\chi) \Omega), \quad \mathcal{T}(\chi)=(\Omega, t(\chi) \Omega),
$$

called transmission and reflection matrices. They obey:

Property 3.3 In each Fock representation of $\left\{\mathcal{C}_{S}, I\right\}, \mathcal{T}(\chi)$ and $\mathcal{R}(\chi)$ satisfy the Hermitian analyticity conditions

$$
\mathcal{R}^{\dagger}(\chi)=\mathcal{R}(-\chi), \quad \mathcal{T}^{\dagger}(\chi)=\mathcal{T}(\chi),
$$


the consistency relations

$$
\begin{aligned}
& \mathcal{S}_{12}\left(\chi_{1}, \chi_{2}\right) \mathcal{R}_{1}\left(\chi_{1}\right) \mathcal{S}_{21}\left(\chi_{2},-\chi_{1}\right) \mathcal{R}_{2}\left(\chi_{2}\right)= \\
& \mathcal{R}_{2}\left(\chi_{2}\right) \mathcal{S}_{12}\left(\chi_{1},-\chi_{2}\right) \mathcal{R}_{1}\left(\chi_{1}\right) \mathcal{S}_{21}\left(-\chi_{2},-\chi_{1}\right) \\
& \mathcal{S}_{12}\left(\chi_{1}, \chi_{2}\right) \mathcal{T}_{1}\left(\chi_{1}\right) \mathcal{S}_{21}\left(\chi_{2}, \chi_{1}\right) \mathcal{T}_{2}\left(\chi_{2}\right)= \\
& \mathcal{T}_{2}\left(\chi_{2}\right) \mathcal{S}_{12}\left(\chi_{1}, \chi_{2}\right) \mathcal{T}_{1}\left(\chi_{1}\right) \mathcal{S}_{21}\left(\chi_{2}, \chi_{1}\right) \\
& \mathcal{S}_{12}\left(\chi_{1}, \chi_{2}\right) \mathcal{R}_{1}\left(\chi_{1}\right) \mathcal{S}_{21}\left(\chi_{2},-\chi_{1}\right) \mathcal{T}_{2}\left(\chi_{2}\right)= \\
& \mathcal{T}_{2}\left(\chi_{2}\right) \mathcal{S}_{12}\left(\chi_{1}, \chi_{2}\right) \mathcal{R}_{1}\left(\chi_{1}\right) \mathcal{S}_{21}\left(\chi_{2},-\chi_{1}\right)
\end{aligned}
$$

and unitarity conditions

$$
\mathcal{T}(\chi) \mathcal{T}(\chi)+\mathcal{R}(\chi) \mathcal{R}(-\chi)=\mathbb{I}, \quad \mathcal{T}(\chi) \mathcal{R}(\chi)+\mathcal{R}(\chi) \mathcal{T}(-\chi)=0 .
$$

Moreover, the vacuum state $\Omega$ is unique (up to a phase factor) and satisfies

$$
r(\chi) \Omega=\mathcal{R}(\chi) \Omega, \quad t(\chi) \Omega=\mathcal{T}(\chi) \Omega .
$$

We thus recover at the level of Fock representation the well-known boundary Yang-Baxter equation (3.17), together with a transmission (3.18) and a transmission-reflection (3.19) Yang-Baxter equation.

\section{Kinematics and RT algebras}

The goal of this section is to present a diagrammatic framework for integrable models with impurity, which sustains the RT algebra presentation. We parameterize the asymptotic particles by spectral parameter $k \in \mathbb{R}$ and an "isotopic" index $i=1, \ldots, N$, corresponding to the auxiliary space. We will use as fundamental building blocks the two-body bulk scattering matrix $S_{12}\left(k_{1}, k_{2}\right)=S_{i_{1} i_{2}}^{j_{1} j_{2}}\left(k_{1}, k_{2}\right) E_{i_{1} j_{1}} \otimes E_{i_{2} j_{2}}$; the reflection matrix $R(k)$, describing the reflection of a particle from the impurity and the transmission matrix $T(k)$, describing the transmission of a particle by the impurity.

It is worth stressing that $S$ is allowed to depend on $k_{1}$ and $k_{2}$ separately 6. 9, 10. Allowing $S$ to depend on $k_{1}$ and $k_{2}$ separately, leads to a natural generalization of the inverse scattering method, which avoids the no-go theorem of [8] and describes a large set of integrable systems, not covered there. We will come back on this point in sect. 6.

It is convenient to define at this stage the matrices

$$
R^{ \pm}(k) \equiv \theta( \pm k) R(k), \quad T^{ \pm}(k) \equiv \theta( \pm k) T(k)
$$

which have a simple physical interpretation: $R^{ \pm}$describe the reflection of a particle propagating $\mathbb{R}_{ \pm}$, whereas $T^{ \pm}$correspond to the transmission of a particle from $\mathbb{R}_{-}$to $\mathbb{R}_{+}$and vice versa, see fig. 1 . Time is flowing along 

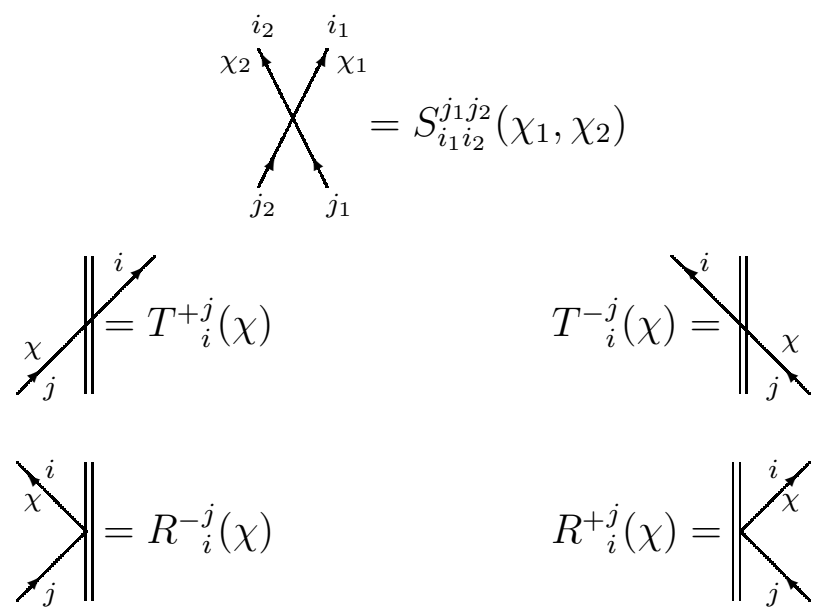

Figure 1: The two-body processes.

the vertical direction and single lines represent particles. The double line corresponds to the impurity world line.

The construction of the possible three-body processes in terms of $S, R^{ \pm}$ and $T^{ \pm}$leads to a series of relations [7, 8. Considering the scattering of three particles, we get the well-known quantum Yang-Baxter equation (in its braid form). The consistency conditions implied by the scattering of two particles among each other and with the impurity are organized in the following three groups:

(a) pure reflection (fig. 2):

$$
\begin{aligned}
& S_{12}\left(k_{1}, k_{2}\right) R_{2}^{+}\left(k_{1}\right) S_{12}\left(k_{2},-k_{1}\right) R_{2}^{+}\left(k_{2}\right)= \\
& R_{2}^{+}\left(k_{2}\right) S_{12}\left(k_{1},-k_{2}\right) R_{2}^{+}\left(k_{1}\right) S_{12}\left(-k_{2},-k_{1}\right), \\
& S_{12}\left(k_{1}, k_{2}\right) R_{1}^{-}\left(k_{2}\right) S_{12}\left(-k_{2}, k_{1}\right) R_{1}^{-}\left(k_{1}\right)= \\
& R_{1}^{-}\left(k_{1}\right) S_{12}\left(-k_{1}, k_{2}\right) R_{1}^{-}\left(k_{2}\right) S_{12}\left(-k_{2},-k_{1}\right) .
\end{aligned}
$$

Eqs. (4.2) and (4.3) concern the reflection on $\mathbb{R}_{+}$and $\mathbb{R}_{-}$respectively. Using the rules of fig. 1, one gets from eq. (4.2) the fig. 2. To any figure, one associates a new one, deduced by reflection with respect to the impurity world line: eq. (4.3) gives the reflection of fig. 2 .

(b) pure transmission (fig. 3):

$$
\begin{aligned}
& T_{1}^{+}\left(k_{1}\right) S_{12}\left(k_{1}, k_{2}\right) T_{1}^{-}\left(k_{2}\right)=T_{2}^{-}\left(k_{2}\right) S_{12}\left(k_{1}, k_{2}\right) T_{2}^{+}\left(k_{1}\right), \\
& S_{12}\left(\chi_{1}, \chi_{2}\right) T_{1}^{-}\left(\chi_{2}\right) T_{2}^{-}\left(\chi_{1}\right)=T_{1}^{-}\left(k_{1}\right) T_{2}^{-}\left(k_{2}\right) S_{12}\left(k_{1}, k_{2}\right), \\
& S_{12}\left(\chi_{1}, \chi_{2}\right) T_{1}^{+}\left(\chi_{2}\right) T_{2}^{+}\left(\chi_{1}\right)=T_{1}^{+}\left(k_{1}\right) T_{2}^{+}\left(k_{2}\right) S_{12}\left(k_{1}, k_{2}\right),
\end{aligned}
$$




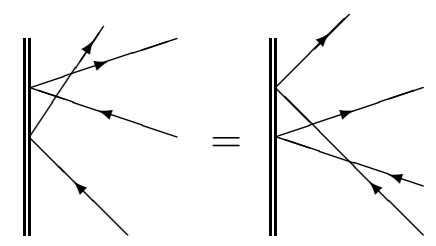

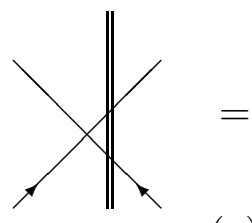

(a)

Figure 2: Pure reflection.

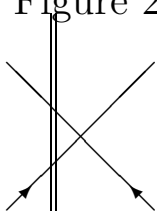

$\nearrow$

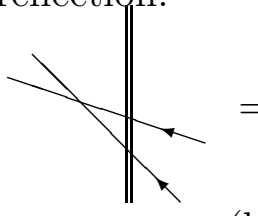

(b)

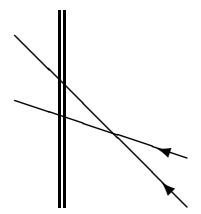

Figure 3: Pure transmission.

(c) mixed relations (fig. 4):

$$
\begin{aligned}
& R_{1}^{+}\left(k_{1}\right) T_{2}^{-}\left(k_{2}\right)=T_{2}^{-}\left(k_{2}\right) S_{12}\left(k_{1}, k_{2}\right) R_{2}^{+}\left(k_{1}\right) S_{12}\left(k_{2},-k_{1}\right), \\
& T_{1}^{+}\left(k_{1}\right) R_{2}^{-}\left(k_{2}\right)=T_{1}^{+}\left(k_{1}\right) S_{12}\left(k_{1}, k_{2}\right) R_{1}^{-}\left(k_{2}\right) S_{12}\left(-k_{2}, k_{1}\right), \\
& R_{1}^{+}\left(k_{1}\right) T_{2}^{+}\left(k_{2}\right)=S_{12}\left(k_{1}, k_{2}\right) R_{2}^{+}\left(k_{1}\right) S_{12}\left(k_{2},-k_{1}\right) T_{2}^{+}\left(k_{2}\right), \\
& T_{1}^{-}\left(k_{1}\right) R_{2}^{-}\left(k_{2}\right)=S_{12}\left(k_{1}, k_{2}\right) R_{1}^{-}\left(k_{2}\right) S_{12}\left(-k_{2}, k_{1}\right) T_{1}^{-}\left(k_{1}\right), \\
& R_{1}^{+}\left(k_{1}\right) T_{2}^{-}\left(k_{2}\right) S_{12}\left(-k_{1}, k_{2}\right)=T_{2}^{-}\left(k_{2}\right) S_{12}\left(k_{1}, k_{2}\right) R_{2}^{+}\left(k_{1}\right), \\
& T_{1}^{+}\left(k_{1}\right) R_{2}^{-}\left(k_{2}\right) S_{12}\left(k_{1},-k_{2}\right)=T_{1}^{+}\left(k_{1}\right) S_{12}\left(k_{1}, k_{2}\right) R_{1}^{-}\left(k_{2}\right), \\
& R_{2}^{+}\left(k_{1}\right) S_{12}\left(k_{2},-k_{1}\right) T_{2}^{+}\left(k_{2}\right)=S_{12}\left(k_{2}, k_{1}\right) R_{1}^{+}\left(k_{1}\right) T_{2}^{+}\left(k_{2}\right), \\
& R_{1}^{-}\left(k_{2}\right) S_{12}\left(-k_{2}, k_{1}\right) T_{1}^{-}\left(k_{1}\right)=S_{12}\left(k_{2}, k_{1}\right) T_{1}^{-}\left(k_{1}\right) R_{2}^{-}\left(k_{2}\right) .
\end{aligned}
$$

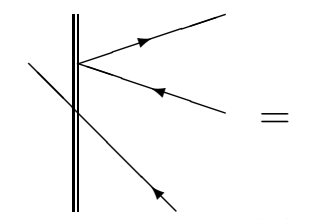

(a)

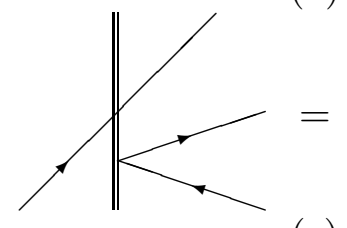

(c)
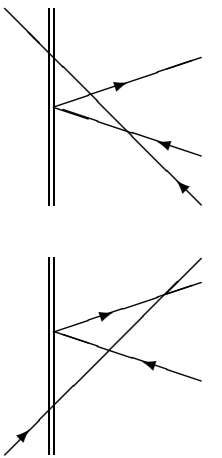
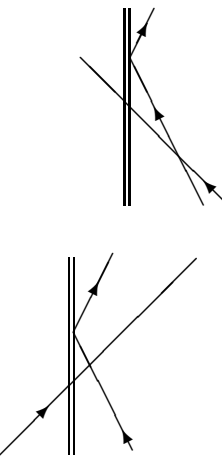

(b)

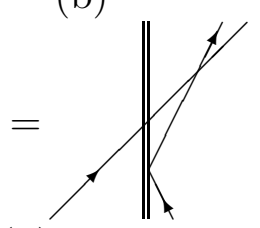

(d)

Figure 4: Mixed relations.

Finally, the requirements of unitarity and Hermitian analyticity lead for $S$ to the familiar [3, 4, 5] conditions (3.12). Concerning $R$ and $T$, these 
conditions read

$$
\begin{aligned}
T(k) T(k)+R(k) R(-k)=1, & T(k) R(k)+R(k) T(-k)=0, \\
{[T]^{\dagger}(k)=T(k), } & {[R]^{\dagger}(k)=R(-k), }
\end{aligned}
$$

The two sets of eqs. (4.7)-(4.10) and (4.11)-(4.14) being equivalent, one is left with the study eqs. (4.24.10) and (4.15)-(4.16).

The impurity at $x=0$ breaking translation invariance, we equip the generators of our algebra with a double index $\alpha=(\xi, i)$, which amounts to extend the auxiliary space from $\operatorname{End}\left(\mathbb{C}^{N}\right)$ to $\operatorname{End}\left(\mathbb{C}^{2 N}\right)$. The index $\xi= \pm$ indicates the half line $\mathbb{R}_{ \pm}$where the particle is created or annihilated. With this notation, we consider an associative algebra $\mathcal{C}$ with identity element $\mathbf{1}$, generated by $\left\{a(k), a^{\dagger}(k)\right\}$, which are subject to the constraints:

$$
\begin{aligned}
& a_{1}\left(k_{1}\right) a_{2}\left(k_{2}\right)-\mathcal{S}_{21}\left(k_{2}, k_{1}\right) a_{2}\left(k_{2}\right) a_{1}\left(k_{1}\right)=0, \\
& a_{1}^{\dagger}\left(k_{1}\right) a_{2}^{\dagger}\left(k_{2}\right)-a_{2}^{\dagger}\left(k_{2}\right) a_{1}^{\dagger}\left(k_{1}\right) \mathcal{S}_{21}\left(k_{2}, k_{1}\right)=0, \\
& a_{1}\left(k_{1}\right) a_{2}^{\dagger}\left(k_{2}\right)-a_{2}^{\dagger}\left(k_{2}\right) \mathcal{S}_{12}\left(k_{1}, k_{2}\right) a_{1}\left(k_{1}\right)= \\
& \quad \delta\left(k_{1}-k_{2}\right)\left[\delta_{12}+\mathcal{T}_{12}\left(k_{1}\right)\right] \mathbf{1}+\delta\left(k_{1}+k_{2}\right) \mathcal{R}_{12}\left(k_{1}\right) \mathbf{1} .
\end{aligned}
$$

The constraints on the matrices $\mathcal{R}(k)$ and $\mathcal{T}(k)$ are given by (3.9)-(3.11). When $\mathcal{T}$ is invertible, they imply [9] the relations (4.2)-(4.10).

Then, the set $\{\mathcal{S}, \mathcal{R}, \mathcal{T}\}$ is related to the starting data $\{S, R, T\}$ of an RT algebra in the following way:

$$
\begin{array}{r}
\mathcal{S}_{\left(\xi_{1}, i_{1}\right)\left(\xi_{2}, i_{2}\right)}^{\left(\eta_{1}, j_{1}\right)\left(\eta_{2}, j_{2}\right)}\left(k_{1}, k_{2}\right) \equiv \delta_{\xi_{1}}^{\eta_{2}} \delta_{\xi_{2}}^{\eta_{1}} S_{i_{1} i_{2}}^{j_{2} j_{1}}\left(k_{1}, k_{2}\right), \\
\mathcal{R}_{(\xi, i)}^{(\eta, j)}(k) \equiv \delta_{\xi}^{\eta} R_{i}^{j}(k), \quad \mathcal{T}_{(\xi, i)}^{(\eta, j)}(k) \equiv \delta_{-\xi}^{\eta} T_{i}^{j}(k),
\end{array}
$$

where the auxiliary space indices have been explicited.

\section{From ZF to RT algebras}

We present here a connection between the ZF algebras and the RT algebras, in the same way boundary algebras (defined for integrable systems on the half-line [6, 14]) can be constructed from ZF algebras [16]. We remind that a $\mathrm{ZF}$ algebras $\mathcal{A}_{S}$ is the polynomial algebra generated by a unit $\mathbf{1}$ and the generators $a(k)$ and $a^{\dagger}(k)$, subject to:

$$
\begin{aligned}
a_{1}\left(k_{1}\right) a_{2}^{\dagger}\left(k_{2}\right) & =a_{2}^{\dagger}\left(k_{2}\right) S_{12}\left(k_{1}, k_{2}\right) a_{1}\left(k_{1}\right)+\delta_{12} \delta\left(k_{1}-k_{2}\right) \mathbf{1} \\
a_{1}\left(k_{1}\right) a_{2}\left(k_{2}\right) & =S_{21}\left(k_{2}, k_{1}\right) a_{2}\left(k_{2}\right) a_{1}\left(k_{1}\right) \\
a_{1}^{\dagger}\left(k_{1}\right) a_{2}^{\dagger}\left(k_{2}\right) & =a_{2}^{\dagger}\left(k_{2}\right) a_{1}^{\dagger}\left(k_{1}\right) S_{21}\left(k_{2}, k_{1}\right)
\end{aligned}
$$

We employ below an extension $\overline{\mathcal{A}}_{S}$ of $\mathcal{A}_{S}$, involving power series in $a(k)$ and $a^{\dagger}(k)$ whose individual terms preserve the particle number. The concept of extended ZF (EZF) algebra $\overline{\mathcal{A}}_{S}$ is relevant for proving [13]: 


\section{Property 5.1 (Well-bred operators)}

Each $\overline{\mathcal{A}}_{S}$ contains an invertible element $L(k)$ satisfying

$$
\begin{aligned}
L_{1}\left(k_{1}\right) a_{2}\left(k_{2}\right) & =S_{21}\left(k_{2}, k_{1}\right) a_{2}\left(k_{2}\right) L_{1}\left(k_{1}\right) \\
L_{1}\left(k_{1}\right) a_{2}^{\dagger}\left(k_{2}\right) & =a_{2}^{\dagger}\left(k_{2}\right) S_{12}\left(k_{1}, k_{2}\right) L_{1}\left(k_{1}\right) \\
S_{12}\left(k_{1}, k_{2}\right) L_{1}\left(k_{1}\right) L_{2}\left(k_{2}\right) & =L_{2}\left(k_{2}\right) L_{1}\left(k_{1}\right) S_{12}\left(k_{1}, k_{2}\right)
\end{aligned}
$$

Hence, $L(k)$ generates a quantum group $\mathcal{U}_{S} \subset \overline{\mathcal{A}}_{S}$.

$L(k)$, called well-bred operator, is explicitly constructed in [13] and admits a series representation in terms of $a(k)$ and $a^{\dagger}(k)$.

We now turn to the construction of RT algebras from ZF ones. Let $R(k)$ and $T(k)$ be any two matrix functions satisfying

$$
R^{\dagger}(k)=R(-k), \quad T^{\dagger}(k)=T(k),
$$

the unitary conditions (3.11) and the boundary Yang-Baxter equation (3.10). It has been shown in [10] that $R(k)$ and $T(k)$ obey automatically the transmission and reflection-transmission Yang-Baxter eqs. (3.8)-(3.9). Then, starting from the EZF algebra $\overline{\mathcal{A}}_{S}$, one proves [11]:

Property 5.2 Let $t(k)=L(k) T(k) L^{-1}(k)$ and $r(k)=L(k) R(k) L^{-1}(-k)$, where $L(k)$ is the well-bred operator of $\overline{\mathcal{A}}_{S}$ and $R(k)$ and $T(k)$ are defined as above. Then, $a(k), a^{\dagger}(k), t(k)$ and $r(k)$ obey the relations (3.4)-(3.11).

Property 5.3 The map

$$
\sigma\left\{\begin{array}{l}
a(k) \rightarrow \alpha(k)=t(k) a(k)+r(k) a(-k) \\
a^{\dagger}(k) \rightarrow \alpha^{\dagger}(k)=a^{\dagger}(k) t(k)+a^{\dagger}(-k) r(-k)
\end{array}\right.
$$

extends to a homomorphism on $\overline{\mathcal{A}}_{S}$.

The homomorphism $\sigma$ is essential for constructing an RT algebra from EZF. Indeed, we have:

\section{Theorem 5.4 Let}

$$
\begin{aligned}
A(k) & =\frac{1}{2}(a(k)+\alpha(k))=\frac{1}{2}([1+t(k)] a(k)+r(k) a(-k)) \\
A^{\dagger}(k) & =\frac{1}{2}\left(a^{\dagger}(k)+\alpha^{\dagger}(k)\right)=\frac{1}{2}\left(a^{\dagger}(k)[1+t(k)]+a^{\dagger}(-k) r(-k)\right)
\end{aligned}
$$

Then $A(k), A^{\dagger}(k), t(k)$ and $r(k)$ form a RT algebra.

In this construction, $\mathcal{K}_{S}$ becomes a Hopf coideal in $\mathcal{U}_{S}$.

Note that if one imposes on $R(k)$ the additional relation $R(k) R(-k)=\mathbb{I}$, one recovers the construction [16] of the boundary algebra $\mathcal{B}_{S}$. 


\subsection{Hamiltonians}

One can associate to any ZF algebra a hierarchy of Hamiltonians

$$
H_{Z F}^{(n)}=\int_{\mathbb{R}} d k k^{n} a^{\dagger}(k) a(k), n \in \mathbb{Z}_{+}
$$

They form an Abelian set and admit as symmetry algebra the quantum group generated by the well-bred operators [13]:

$$
\left[H_{Z F}^{(n)}, L(k)\right]=0, \forall n
$$

In a similar way, for any RT algebra, one introduces [11]

$$
H_{R T}^{(n)}=\int_{\mathbb{R}} d k k^{n} A^{\dagger}(k) A(k)
$$

which obey to the following relations:

$$
\left[H_{R T}^{(m)}, H_{R T}^{(n)}\right]=\left[(-1)^{m}-(-1)^{n}\right] \int_{\mathbb{R}} d k k^{m+n} A^{\dagger}(k) r(k) A(-k),
$$

Thus, the Hamiltonians of the same parity define a hierarchy corresponding to integrable systems with impurity.

Property 5.5 The subalgebra $\mathcal{K}_{S}$ is a symmetry algebra of the hierarchy $H_{R T}^{(n)}:\left[H_{R T}^{(n)}, t(k)\right]=0$ and $\left[H_{R T}^{(n)}, r(k)\right]=0$.

This result provides an universal model-independent description of the symmetry content of the hierarchy $H_{R T}^{(n)}$. Remark that $r(k)$ and $t(k)$ encode both the particle-impurity interactions and the quantum integrals of motion. This property of $\mathcal{K}_{S}$ has been applied in [18] for studying the symmetries of the nonlinear Schrödinger equation on the half line.

Let us observe in conclusion that there is a simple relation [11] between the hierarchies with and without impurity:

$$
H_{R T}^{(n)}=H_{Z F}^{(n)}+\int_{\mathbb{R}} d k k^{n} a^{\dagger}(k)(r(k) a(-k)+t(k) a(k))
$$

It extends the results given in [17] in the case of purely reflecting boundary. 


\section{Including an impurity on the line}

It is natural to expect the existence of an integrable system with impurity associated to (almost) any integrable system defined on the whole line. However, the class of integrable systems possessing translation invariant $S$-matrix seems to be excluded [8]. The aim of this section is to show how RT algebras can circumvent this no-go theorem. Starting from an integrable system defined on a line and possessing a translation invariant $S$-matrix, we present here a general construction that allows to obtain an integrable system, associated to the original one, but with an impurity located at the origin which reflects and transmits particles.

Let $s_{12}\left(k_{1}-k_{2}\right) \in \operatorname{End}\left(\mathbb{C}^{N}\right) \otimes \operatorname{End}\left(\mathbb{C}^{N}\right)$ be the translation invariant $S$ matrix of the initial system. From this $S$-matrix, we define a new $S$-matrix:

$$
S\left(k_{1}, k_{2}\right)=\left(\begin{array}{cccc}
s_{12}\left(k_{1}-k_{2}\right) & 0 & 0 & 0 \\
0 & s_{12}\left(k_{1}+k_{2}\right) & 0 & 0 \\
0 & 0 & s_{12}\left(-k_{1}-k_{2}\right) & 0 \\
0 & 0 & 0 & s_{12}\left(k_{2}-k_{1}\right)
\end{array}\right)
$$

which belongs to $\operatorname{End}\left(\mathbb{C}^{2 N}\right) \otimes \operatorname{End}\left(\mathbb{C}^{2 N}\right)$. It is a simple matter of calculation to show that this $S$-matrix also obey the Yang-Baxter equation and unitary condition (3.12). To this $S$-matrix we associate a RT-algebra with generators $t(k), r(k)$ and

$$
A(k)=\left(\begin{array}{c}
a_{+}(k) \\
a_{-}(k)
\end{array}\right), A^{\dagger}(k)=\left(a_{+}^{\dagger}(k), a_{-}^{\dagger}(k)\right)
$$

where $a_{ \pm}(k)$ and $a_{ \pm}^{\dagger}(k)$ are $N$-vectors (column and line respect.), so that $A_{ \pm}(k)$ and $A_{ \pm}^{\dagger}(k)$ are $2 N$-vectors. The defining relations for the RT-algebra are those given in (3.1)-(3.10), with the $S$-matrix (6.1).

The physical interpretation of such a system is clear: the generators $a_{ \pm}(k)$ and $a_{ \pm}^{\dagger}(k)$ correspond to annihilation and creation operators in $\mathbb{R}_{ \pm}$. They interact among themselves with the translation invariant $s_{12}\left(k_{1}-k_{2}\right)$ matrix, in accordance with the naive picture that "far from the impurity", the translation invariance should not be broken. However, the exchange of "+" with "-" operators (which must cross the impurity) is done with the matrix $s_{12}\left(k_{1}+k_{2}\right)$ which is not translation invariant. Consequently, the complete matrix $S\left(k_{1}, k_{2}\right)$ is not translation invariant either, so that the property stated in [8] does not apply here, as it was pointed out in [9]. Note also that despite the translation invariance, the generators $\left\{a_{\xi}(k), a_{\xi}^{\dagger}(k)\right\}(\xi=+$ or -$)$ does not obey ZF algebra relations, but rather boundary algebra ones. This is not surprising, this latter algebra being introduced for systems defined on the half-line $[6$. 
In view of the construction, one can look for generators of the form

$$
t(k)=\left(\begin{array}{cc}
0 & \tau(k) \\
\tau(-k) & 0
\end{array}\right) \text { and } r(k)=\left(\begin{array}{cc}
\rho(k) & 0 \\
0 & \rho(-k)
\end{array}\right)
$$

The defining relations (3.8)-(3.10), expressed for $r(k)$ and $t(k)$ with $S\left(k_{1}, k_{2}\right)$, then become:

$s_{12}\left(k_{1}-k_{2}\right) \tau_{1}\left(k_{1}\right) s_{21}\left(k_{2}-k_{1}\right) \tau_{2}\left(k_{2}\right)=\tau_{2}\left(k_{2}\right) s_{12}\left(k_{1}-k_{2}\right) \tau_{1}\left(k_{1}\right) s_{21}\left(k_{2}-k_{1}\right)$

$s_{12}\left(k_{1}-k_{2}\right) \tau_{1}\left(k_{1}\right) s_{21}\left(k_{2}-k_{1}\right) \rho_{2}\left(k_{2}\right)=\rho_{2}\left(k_{2}\right) s_{12}\left(k_{1}+k_{2}\right) \tau_{1}\left(k_{1}\right) s_{21}\left(-k_{2}-k_{1}\right)$

$s_{12}\left(k_{1}-k_{2}\right) \rho_{1}\left(k_{1}\right) s_{21}\left(k_{2}+k_{1}\right) \rho_{2}\left(k_{2}\right)=\rho_{2}\left(k_{2}\right) s_{12}\left(k_{1}+k_{2}\right) \rho_{1}\left(k_{1}\right) s_{21}\left(k_{1}-k_{2}\right)$

while the unitary relations (3.11) rewrite

$$
\begin{array}{lll}
\tau(k) \tau(-k)+\rho(k) \rho(-k)=\mathbb{I}, & & \tau(k)^{\dagger}=\tau(-k) \\
\tau(k) \rho(-k)+\rho(k) \tau(-k)=0, & & \rho(k)^{\dagger}=\rho(-k)
\end{array}
$$

Note that this presentation has symmetrized the role of $\tau(k)$ and $\rho(k)$.

Finally, let us remark that this construction can obviously be applied to any $S$-matrix, translation invariant or not.

\section{References}

[1] A. Zamolodchikov and A. Zamolodchikov, Factorized S-matrices in two dimensions as the exact solutions of certain relativistic quantum field theory models, Ann. Phys. (N.Y.) 120 (1979) 253.

[2] L.D. Faddeev, Quantum completely integrable models in field theory, Sov. Sci. Rev. C1 (1980) 107.

[3] I. Cherednik, Factorizing particles on a half line and root systems, Theor. Math. Phys. 61 (1984) 977 [Teor. Mat. Fiz. 61 (84) 35].

[4] E.K. Sklyanin, Boundary conditions for integrable quantum systems, J. Phys. A21 (1988) 2375.

[5] S. Ghoshal and A.B. Zamolodchikov, Boundary S-Matrix and Boundary State in Two-Dimensional Integrable Quantum Field Theory, Int. J. Mod. Phys. A9 (1994) 3841 [Erratum, ibid. A9 (1994) 4353], hep-th/9306002.

[6] A. Liguori, M. Mintchev and M. Rossi, Fock Representations Of Exchange Algebras With Involution, J. Math. Phys. 38 (1997) 2888. 
[7] G. Delfino, G. Mussardo and P. Simonetti, Scattering Theory and Correlation Functions in Statistical Models with a Line of Defect, Nucl. Phys. B432 (1994) 518, hep-th/9409076;

R. Konik and A. LeClair, Purely Transmitting Defect Field Theories, Nucl. Phys. B538 (1999) 587, hep-th/9703085.

[8] O.A. Castro-Alvaredo, A. Fring and F. Gohmann, On the absence of simultaneous reflection and transmission in integrable impurity systems, hep-th/0201142.

[9] M. Mintchev, E. Ragoucy and P. Sorba, Scattering in the Presence of a Reflecting and Transmitting Impurity, Phys. Lett. B547 (2002) 313, hep-th/0209052.

[10] M. Mintchev, E. Ragoucy and P. Sorba, Reflection-Transmission Algebras, J. Phys. A36 (2003) 10407, hep-th/0303187.

[11] M. Mintchev and E. Ragoucy, Interplay between ZamolodchikovFaddeev and Reflection-Transmission algebras, J. Phys. A37 (2004) 425, math.QA/0306084.

[12] S. Albeverio, F. Gesztesy, R. Hoegh-Krohn and H. Holden, Solvable models in quantum mechanics (Springer-Verlag, New York, 1988).

[13] E. Ragoucy, Vertex operators for quantum groups and application to integrable systems, J. Phys. A35 (2002) 7929, math.QA/0108207.

[14] A. Liguori, M. Mintchev and L. Zhao, Boundary exchange algebras and scattering on the half-line, Commun. Math. Phys. 194 (1998) 569, hep-th/9607085.

[15] P. Bowcock, E. Corrigan and C. Zambon, Classically integrable field theories with defects, hep-th/0305022.

[16] E. Ragoucy, Vertex operators for boundary algebras, Lett. Math. Phys. 58 (2001) 249, math.QA/0108221.

[17] E. Ragoucy, Quantum group symmetry of integrable systems with or without boundary, Int. J. Mod. Phys. A17 (2002) 3649, math.QA/0202095.

[18] M. Mintchev, E. Ragoucy and P. Sorba, Spontaneous symmetry breaking in the gl(N)-NLS algebra, J. Phys. A34 (2001) 8345, hep-th/0104079. 